\title{
Experimental Study on the Solidification Behaviors in a Latent Heat Thermal Energy Storage Unit with Tree-Shaped Fins
}

\author{
Chengcheng Fan ${ }^{1}$, Hao Xu², Chengbin Zhang ${ }^{1}$, Zilong Deng1 \\ ${ }^{1}$ School of Energy and Environment, Southeast University \\ No.2 Sipailou Avenue Nanjing, Jiangsu, PR China \\ ccfan@microflows.net; xuhaoxt@126.com \\ ${ }^{2}$ School of Environmental Science and Engineering, Suzhou University of Science and Technology \\ No.1 Kerui Road, Suzhou, Jiangsu, PR China \\ cbzhang@aliyun.com; zldeng@microflows.net
}

\begin{abstract}
LHTES unit. A visualization experimental investigation of the solidification process in the LHTES unit with tree-shaped fins is conducted. The dynamic temperature distribution and the solidification front evolution is analysed and compared to those in a corresponding LHTES with plate fins. Additionally, by arranging a certain number of thermocouples at different positions, the temperature changes of the fin region and the PCM region of the tree-shaped structure heat exchanger were recorded during the experiment. Then, the temperature of PCM at different radial distances is analyzed,
\end{abstract}

Keywords: Experimental study, Heat-transfer, Tree-shaped fins, Solidification

\section{Introduction}

Environmental issues and Energy sustainable development are the two critical problems restricting the development of the world today. The vigorous development of sustainable energies has been the consensus of all countries. Although the sustainable energy sources are extensive and the reserves are abundant, the poor efficiency of the energy conversion and utilization delays the process of energy structure adjustment of the word. Moreover, since the intermittent and unstable nature of these sustainable energies, it is necessary to apply an energy storage system to store excess energy to reduce the imbalance between energy demand and supply. Due to the high energy storage density and approximately isothermal energy storage process, latent heat thermal energy storage (LHTES) technology based on phase change material (PCM) has been widely used in the fields of industrial waste heat recovery[1], solar photovoltaic power generation[2], electric power peak-load shifting[3], building energy conservation[4] and spacecraft thermal management[5].

However, most PCMs have the disadvantage of low thermal conductivity, which severely restricts the application and promotion of LHTES technology in practical engineering. So far, many thermal performance enhancement strategies, including the addition of fins, PCM micro-encapsulation, and the addition of high thermal conductivity materials, have been applied to improve the heat storage efficiency of LHTES units. Among them, the addition of fins is most common in practical engineering applications because of easy operation and lower maintenance costs. However, the addition of fins also weakens the heat storage capacity of the LHTES unit to a certain extent. Considering the trade-off between high heat storage capacity and rapid heat storage and release rate, the optimization of fin geometry is of great significance. It is noteworthy that the structure in nature usually has superior heat and mass transfer characteristics because of natural selection. Moreover, the available fin geometry of the traditional LHTES unit is too simple and poor in the spatial layout. Therefore, high attention should be paid to the fin optimization for maximizing the thermal performance of the LHTES unit.

\section{Experimental Descriptions}

The experiment setup is illustrated in Figure 1. The experiment system includes tree-shaped fins heat exchanger, data acquisition system and temperature control system. As shown in the Fig.1, the Tree-shaped fins heat exchange mainly includes Tree-shaped fins, PCM, Casing, Shell and Cover. The aluminum 6061 is chosen as the solid material and lauric acid 
is selected as PCM in this paper. During the experiment, the hot fluid in the temperature control system controls the flow through the three-way valve and the flow meter. The phase change material filled in the snowflake fractal heat

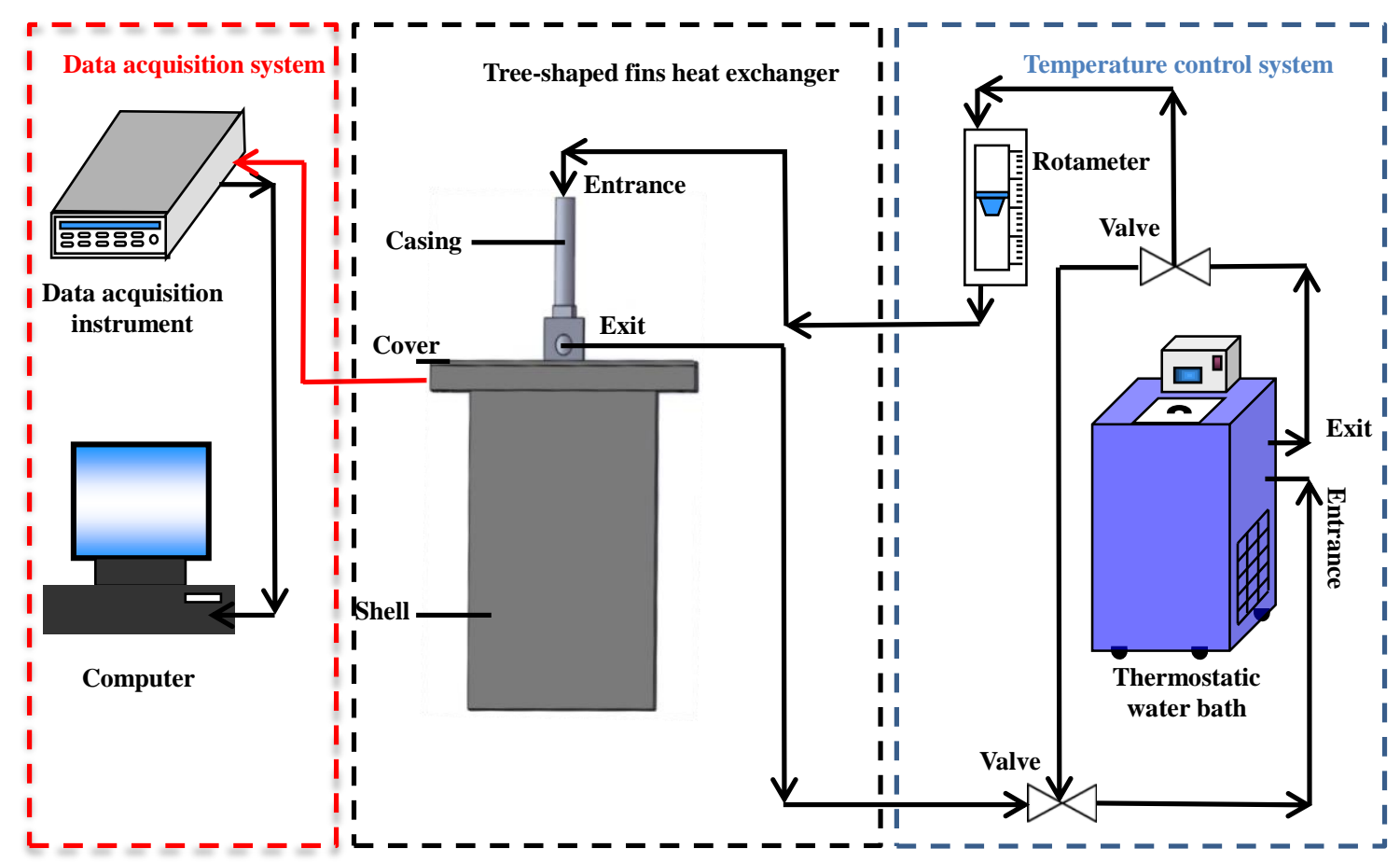

Fig.1: Experimental setup

exchanger can absorb heat from the fluid in the sleeve and phase change through the snowflake fractal structure The thermocouple inside the heat exchanger can sense the temperature change inside the heat exchanger in order to further analysis the heat transfer performance of the tree-shaped fins phase change heat exchanger. The temperature control system is mainly composed of a constant temperature water bath, a rotor flow meter and a control valve. The model of the thermostatic water bath is RW-3400G, made in Korea by Jeiotech.
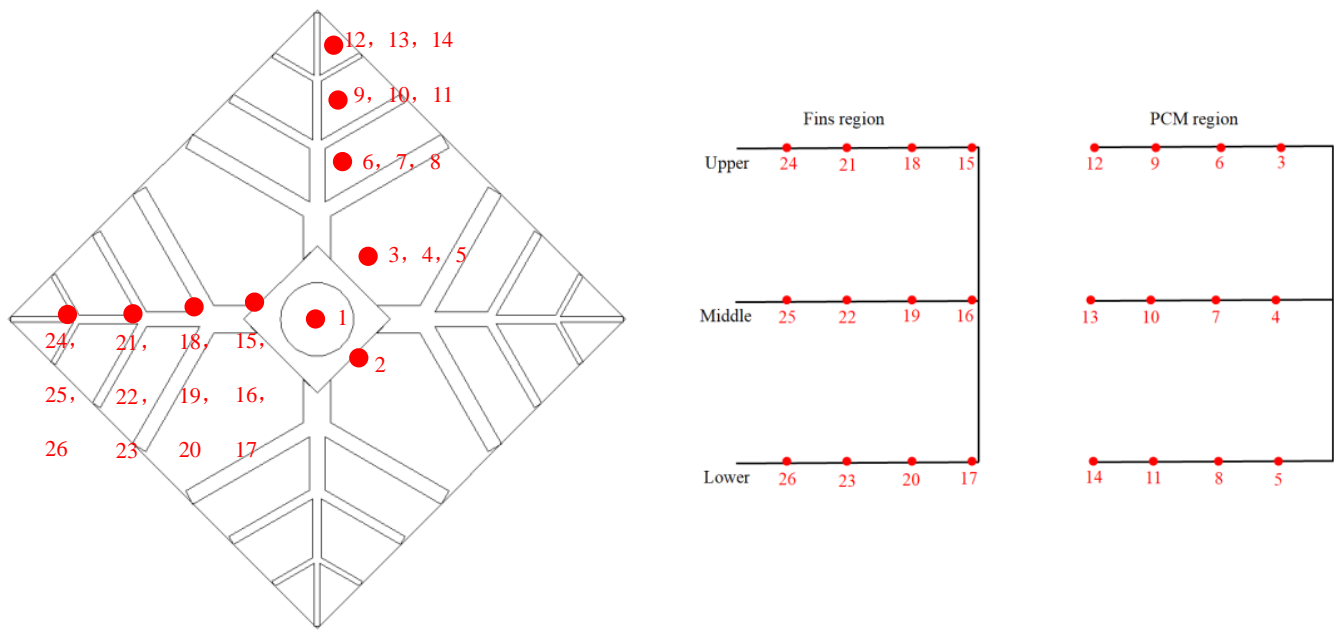

Fig.2: Schematic diagram of thermocouple arrangement 
In this experiment, thermocouples were arranged in the fin region, PCM region of the tree-shaped fins LHTES, and the water inlet and outlet of the bushing. The layout of the thermocouple is shown in Fig.2. As can be seen from the figure, a total of four thermocouple measuring points are arranged on the LHTES, of which No.15 measuring points are located on the trunk, No.18, 21, 24 measuring points are located on the branch, and two thermocouples are arranged on the wall of tube. No.1 measuring point is near the entrance, No.2 measuring point are near the exit. PCM area has a total of twelve thermocouple measurement points, of which 3, 6, 9, 12 are near the top, 4, 7, 10, 13 is located in the middle and the measuring points of $5,8,11,14$ are close to the bottom. The vertical position is consistent with the position of the fin system for comparison and analysis. The centralized temperature expression at each position is shown in Equation (1)-(6).

$$
\begin{gathered}
T_{\text {outside }}=\frac{T_{3}+T_{4}+T_{5}}{3} \\
T_{\text {middle_R }}=\frac{T_{6}+T_{7}+T_{8}+T_{9}+T_{10}+T_{11}}{6} \\
T_{\text {inside }}=\frac{T_{3}+T_{4}+T_{5}}{3} \\
T_{\text {upper }}=\frac{T_{3}+T_{6}+T_{9}+T_{12}}{4} \\
T_{\text {middle_A }}=\frac{T_{4}+T_{7}+T_{10}+T_{13}}{4} \\
T_{\text {lower }}=\frac{T_{5}+T_{8}+T_{11}+T_{14}}{4}
\end{gathered}
$$

\section{Results and Discussions}

\subsection{Thermal Performance in the Axial Direction}

During the solidification process, the temperature change curve has undergone the following four processes. The first is that the liquid PCM transfers sensible heat to the metal rib, and the metal rib transmits heat to the heat transfer medium in the pipe. The entire process mainly releases PCM's Sensible heat so that the temperature change rate is relatively fast. Second stage is the PCM solidification processing, because PCM will release a large amount of latent heat during the solidification process, which makes the temperature stable. PCM also changes from liquid to the two-phase mixed status to the solid state. Then, after the PCM is solidified, the heat is transferred again as sensible heat, and the temperature in each region also drops again. Finally, when the temperature in each region stabilizes, the entire heat exchange system reach thermal equilibrium. To further understand the thermal performance enhancement, the solidification processing of LHTS units with two different direction. Fig.3 illustrates the temperature variation of different layer in the axial direction. The solidification process is mainly led by heat conduction, and the temperature change at the inlet and outlet of the axial heat exchange fluid is small (maximum difference is $4^{\circ} \mathrm{C}$ ), so the axial temperature uniformity is better. 


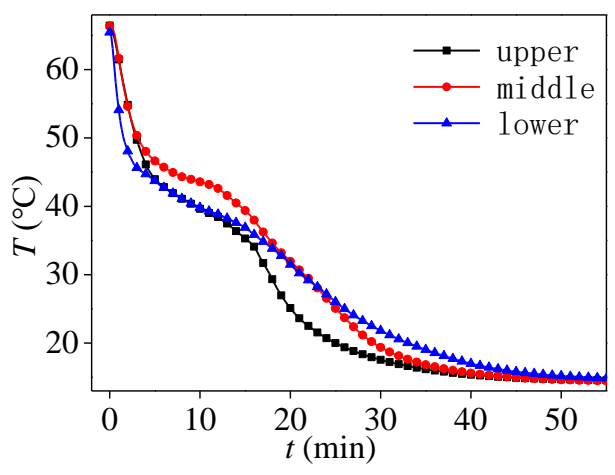

Fig.3: Axial solidification processing

\subsection{Thermal Performance in the Radial Direction}

Generally speaking, organic PCM have the disadvantage of low thermal conductivity. In this solidification heat transfer experiment, the tree-shaped structure was designed to increase the heat transfer area and strengthen the speed and efficiency of heat transfer, thereby improving the entire heat transfer performance of heat exchangers. Fig.5 shows the time-varying curves of the radial temperatures of the PCM different layers in the tree-shaped fractal structure heat exchanger. It can be found that the outside PCM has no obvious phase change process. This is because the outside PCM is close to the outer boundary, and the latent heat released during the solidification process will be partially dissipated, making the entire process sensible heat dominating. There is no obvious 'phase change step'. In addition, the rate of heat transfer by metal fins is much higher than that of PCM, so that at the same time, the temperature difference between the edge of the metal fin and the middle of the fin is very small, and the heat transferred to the PCM is not much different.

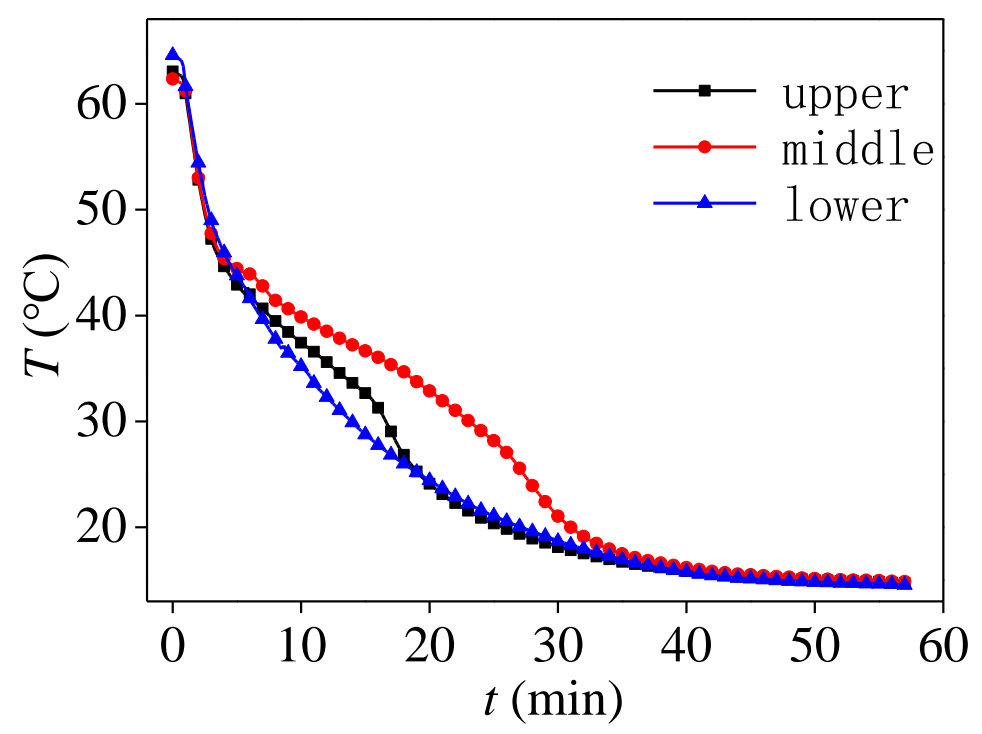

(a)

Fig.5 Radial solidification processing 


\section{Conclusion}

In this paper, a tree-shaped structure LHTS unit is designed, and a solidification phase change heat transfer experiment experiment platform was set up. Thermocouples are respectively arranged in the metal fin region and the PCM region of the of the heat exchanger. The dynamic temperature of the specific position of the fractal structure heat exchanger is monitored monitored in order to study the relevant laws of the entire heat exchanger during the phase change heat transfer process, and the following conclusions are obtained.

When PCM is added to the tree fractal structure heat exchanger, it can be found that during the phase change process, the latent heat of PCM has become an important part of the total heat energy. Reasonable use of the phase change latent heat of PCM can effectively improve the heat transfer performance of the heat exchanger.

From radial angle, during the solidification process, the temperature difference between the PCM near the outermost layer and the PCM close to center in the heat exchanger is not much different. On the one hand, the radial distance is shorter than the axial distance, and the heat transfer is fast, making the temperature uniformity of the entire solidification process better. On the other hand, the design of the tree-shaped fractal structure metal fins provides fast heat diffusion channels from points and surfaces, so that the heat transfer in each area is more uniform.

\section{References}

[1] K. Merlin, J. Soto, D. Delaunay, L. Traonvouez, Industrial waste heat recovery using an enhanced conductivity latent heat thermal energy storage, Appl. Energy 183 (2016) 491-503.

[2] Y.Tian, C.Y. Zhao, A review of solar collectors and thermal energy storage in solar thermal applications, Appl. Energy 104 (2013) 538-553.

[3] Y.j. Sun, S.W. Wang, F. Xiao, D.C. Gao, Peak load shifting control using different cold thermal energy storage facilities in commercial buildings: A review, Appl. Energy 71 (2013) 101-114.

[4] A.M. Khudhair, M.M. Farid, A review on energy conservation in building applications with thermal storage by latent heat using phase change materials, Energy Convers. Manage. 45(2) (2004) 263-275.

[5] J.C. Mulligan, D.P. Colvin, Y.G. Bryant, Microencapsulated phase-change material suspensions for heat transfer in spacecraft thermal systems, J. Spacecraft Rockets 33(2) (1996) 278-291. 